\title{
CLASSES OF PLUS MATRICES IN FINITE DIMENSIONAL INDEFINITE SCALAR PRODUCT SPACES
}

\author{
Cornelis V. M. van der Mee, ${ }^{1}$ André C.M. Ran and L. Rodman ${ }^{2}$
}

Dedicated to M.G. Krein, one of the founding fathers of the theory of plus-operators.

We describe in terms of canonical forms various classes of plus matrices in real and complex finite dimensional spaces with indefinite scalar product and study their topological structures (such as closure and interior).

\section{Introduction}

Let $F$ be the field of real numbers $\mathbf{R}$ or the field of complex numbers $\mathbf{C}$. Choose a fixed real symmetric (if $F=\mathbf{R}$ ) or complex hermitian (if $F=\mathbf{C}$ ) invertible $n \times n$ matrix $H$. Consider the scalar product induced by $H$ by the formula $[x, y]=\langle H x, y\rangle, x, y \in F^{n}$. Here $\langle\cdot, \cdot\rangle$ stands for the usual scalar product in $F^{n}$, i.e.,

$$
\langle x, y\rangle=\sum_{j=1}^{n} x_{j} \overline{y_{j}}
$$

where $x$ and $y$ are the column vectors with components $x_{1}, \cdots, x_{n}$ and $y_{1}, \cdots, y_{n}$, respectively, and $\overline{y_{j}}=y_{j}$ if $F=\mathbf{R}$. The scalar product $[\cdot, \cdot]$ is nondegenerate $\left([x, y]=0\right.$ for all $y \in F^{n}$ implies $x=0$ ), but is indefinite in general. The vector $x \in F^{n}$ is called positive if $[x, x]>0$, neutral if $[x, x]=0$, and negative if $[x, x]<0$.

Well-known concepts related to scalar products are defined here in an obvious way. Thus, given an $n \times n$ matrix $A$ over $F$, the $H$-adjoint $A^{[*]}$ is defined by $[A x, y]=\left[x, A^{[*]} y\right]$ for all $x, y \in F^{n}$. In that case $A^{[*]}=H^{-1} A^{*} H$, where $A^{*}$ denotes the conjugate transpose of $A$ (with $A^{*}=A^{T}$, the transpose of $A$, if $F=\mathbf{R}$ ). An $n \times n$ matrix $A$ is called

${ }^{1}$ The work of this author was performed under the auspices of C.N.R.-G.N.F.M. and partially supported by the research project, "Nonlinear problems in analysis and its physical, chemical and biological applications: Analytical, modelling and computational aspects," of the Italian Ministry of Higher Education and Research (M.U.R.S.T.)

${ }^{2}$ The work of this author partially supported by the NSF grant DMS 9500924 .

The work of all authors is partially supported by the NATO grant CGR 960700 . 
$H$-selfadjoint if $A^{[*]}=A$ (or equivalently, if $H A$ is hermitian). An $n \times n$ matrix $U$ is called $H$-unitary if $[U x, U y]=[x, y]$ for all $x, y \in F^{n}$ (or equivalently, if $U^{*} H U=H$ ).

An $n \times n$ matrix $X$ (over $F$ ) will be called an $H$-plus matrix if $\left[X^{[*]} X u, u\right]=[X u, X u] \geq 0$ whenever $[u, u] \geq 0$. Clearly, for an $H$-plus matrix $X$ we have $\left[X^{[*]} X u, u\right] \geq 0$ whenever $[u, u]>0$. Thus defining

$$
\mu(X)=\inf _{[u, u]=1}\left[X^{[*]} X u, u\right]
$$

we see that $X$ is an $H$-plus matrix if and only if $\mu(X) \geq 0$ (it is assumed here that the set of vectors $u$ such that $[u, u]=1$ is not empty, i.e., $H$ is not negative definite). Then (see [Bo], Theorem II 8.1 in the complex case, and [BMRRR2] in the real case)

$$
\left[X^{[*]} X z, z\right] \geq \mu(X)[z, z], \quad z \in F^{n} .
$$

We call $X$ a strict $H$-plus matrix if $\mu(X)>0$. The matrix $X$ is called a doubly $H$-plus matrix if both $X$ and $X^{[*]}$ are $H$-plus matrices. Throughout the paper we denote by $\mathcal{P}$ the set of $H$-plus matrices, by $\mathcal{S P}$ the set of strict $H$-plus matrices, and by $\mathcal{D P}$ the set of all doubly $H$-plus matrices (the dependence of these classes on $H$ and on $F$ is suppressed in this notation). Another class of interest is the set of strongly strict $H$-plus matrices. A matrix $X$ will be called a strongly strict $H$-plus matrix (notation $X \in \mathcal{S S P}$ ) if $[u, u] \geq 0, u \neq 0$, implies that $[X u, X u]>0$. In [IKL] the terminology " $B$-plus operators" is used to describe the $\mathcal{S S P}$ property (in the context of operators in Pontryagin spaces); the class of $B$-plus operators was introduced in $[\mathrm{Br}]$.

If $H$ is positive definite, every $n \times n$ matrix is an $H$-plus matrix and $\mu(X)$ is the smallest (automatically nonnegative) eigenvalue of $X^{[*]} X$. Then $X$ is a strict $H$-plus matrix if and only if it is invertible. On the other hand, if $H$ is negative definite, every $n \times n$ matrix $X$ is an $H$-plus matrix. In the rest of this paper we will therefore assume that $H$ is indefinite.

Linear operators on a Hilbert space with indefinite scalar product that transform nonnegative vectors into nonnegative vectors, have been studied extensively by Krein and Shmul'jan [KS1,KS2], who called them "plus operators." The various connections with nonexpansive linear operators [Gi1, Gi2], the $J$-modulus [P2], $J$-contractions and $J$-expansions ([P1]; also [BMRRR2]) as well as the theory of plus operators themselves, including the classes $\mathcal{S P}$, $\mathcal{D P}$ and $\mathcal{S} \mathcal{S P}$, have been discussed in various textbooks [Bo, IKL, AI]. These results, however, are formulated in an infinite dimensional setting and hence the term "plus operator" is appropriate. In the present article, as well as in [BMRRR2], we are working exclusively in a finite dimensional context and hence we adopt the term " $H$-plus matrix" instead.

Recently there has been a flurry of activity on plus operators in a finite dimensional context (" $H$-plus matrices") and its applications to linear optics and relativity theory. In linear optics the input-output map can be represented by a real $4 \times 4$ matrix, the so-called Mueller matrix, which is an $H$-plus matrix for the case when $F=\mathbf{R}, n=4$ and $H=$ $\operatorname{diag}(1,-1,-1,-1)([\mathrm{M}, \mathrm{MH}])$. In this case the $(1,1)$-element must be nonnegative and the $H$-adjoint must also be an $H$-plus matrix. Another application of $H$-plus matrices (with exactly the same $F, n$ and $H$ ) is relativity theory, where the linear transformations leaving invariant the sets of time-like and light-like vectors in Minkowski space are $H$-plus matrices.

The applications to linear optics and relativity theory involve the matrices leaving invariant the set of vectors $\left(x_{1}, x_{2}, x_{3}, x_{4}\right) \in \mathbf{R}^{4}$ such that $x_{1} \geq\left(x_{2}^{2}+x_{3}^{2}+x_{4}^{2}\right)^{1 / 2}$. This set is a positive cone in $\mathbf{R}^{4}$ and hence the matrices leaving it invariant can be studied alternatively using the Perron-Frobenius theory of positive matrices $[\mathrm{Ba}, \mathrm{BP}]$. The obvious generalization 
of this cone to $\mathbf{R}^{n}$ is usually called the ice-cream cone (see, e.g., [LS]). Such additional structure of $H$-plus matrices exists if $H$ has only one positive eigenvalue. For more general $H$ the theory of $H$-plus matrices is much more complicated.

Another recent development relevant to $H$-plus matrices stems from the successful attempt to generalize the concepts of norm, spectral radius and singular numbers to $F^{n}$ equipped with an indefinite scalar product [BG1, BG2]. There turns out to be a close connection between the $G$-bound first introduced in [BG1] and strict $H$-plus matrices.

A third recent development involves the characterization of the matrices $X$ with given $X^{[*]} X$ (i.e., with given $X^{*} H X$ ) [BR] and the study of $H$-polar decompositions, i.e., representations of $X$ of the form $X=U A$ where $U$ is $H$-unitary and $A$ is $H$-selfadjoint [BR, BMRRR1, BMRRR2]. Partial results involving the $J$-modulus [P2] could be generalized and interesting special cases, like those of $H$-plus and strict $H$-plus matrices, were discussed. In particular, characterizations of $H$-plus and strict $H$-plus matrices in terms of the canonical form of the pair of matrices $\left(X^{[*]} X, H\right)$ were given in [BMRRR2].

In the present paper we focus exclusively on $H$-plus matrices. We will solve the following problems:

(a) Characterization of $\mathcal{S S P}, \mathcal{S P}, \mathcal{D P}$ and $\mathcal{P}$ and other classes of $H$-plus matrices.

(b) Determination of the interior and the closure of the various classes of $H$-plus matrices. Here, the description of the closure of $\mathcal{S P}$ is still an open problem in general.

(c) Specialization of the results for the ice-cream cone, i.e., for the case $F=\mathbf{R}$ and $H=\operatorname{diag}(1,-1, \cdots,-1)$.

In contrast to the previous works on the class of plus operators and its various subsets (with the exception of [BMRRR2]) the main tool in our investigation is the canonical form of selfadjoint matrices in finite dimensional spaces with an indefinite scalar product, which is recalled in section 2 . We consider the real case as well as the complex case; the previous works (again with the exception of [BMRRR2]) focused on the complex case.

In Section 2 we introduce the characterizations of the various classes of $H$-plus matrices, alongside basic definitions and illustrative examples. In Section 3 we study some of the topological properties of $\mathcal{P}, \mathcal{S P}, \mathcal{S S P}$ and $\mathcal{D P}$. More precisely, we determine their interiors, closures and connectivity properties at least partially. For the ice-cream cone complete results are obtained in Section 4. In this section we also introduce the ice-cream cone itself and specialize our other results to this case.

The following notations will be used. The subspace of all eigenvectors and generalized eigenvectors of a matrix $X$ corresponding to the eigenvalue $\lambda$, together with the zero vector, is denoted by $R_{\lambda}(X)$. The block diagonal matrix with matrices $Z_{1}, \ldots, Z_{k}$ on the diagonal is denoted by $Z_{1} \oplus \cdots \oplus Z_{k}$. The set of eigenvalues (including the nonreal eigenvalues for real matrices) of a matrix $X$ is denoted by $\sigma(X)$. $\operatorname{Ker} A$ and $\operatorname{Im} A$ stand for the null space and range of a matrix $A$, respectively.

Unless indicated otherwise, the results of Sections 2 and 3 are valid for both the real and the complex cases. 


\section{Characterizations in Terms of Canonical Forms}

In this section we characterize the classes $\mathcal{P}$ of $H$-plus matrices, $\mathcal{S P}$ of strict $H$-plus matrices, and $\mathcal{S S P}$ of strongly strict $H$-plus matrices in terms of the canonical form of the pair of matrices $\left(X^{[*]} X, H\right)$ and give a complete description of certain related classes of matrices. Before stating and proving these characterizations we define the canonical form of a pair of $n \times n$ matrices $(A, H)$ where $H$ is an invertible selfadjoint matrix and $A$ is $H$-selfadjoint.

Let $J_{k}(\lambda)$ denote the $k \times k$ upper triangular Jordan block with $\lambda \in \mathbf{C}$ on the diagonal and let $J_{k}(\lambda \pm i \mu)$ denote the matrix

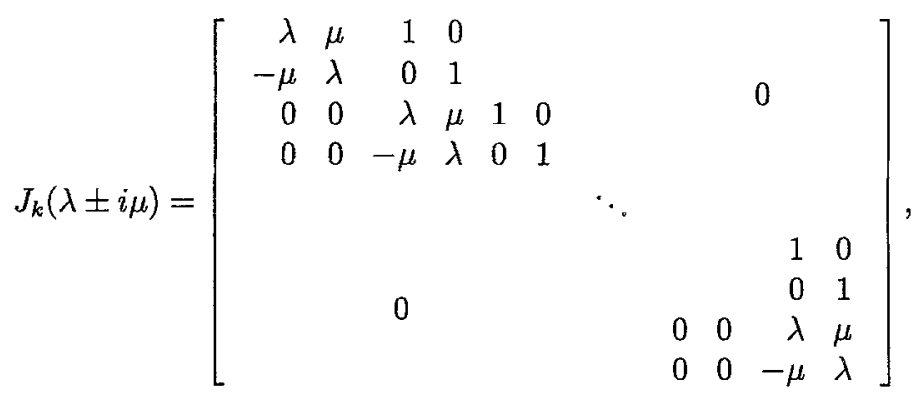

where $\lambda, \mu \in \mathbf{R}, \mu>0$ and $k$ is necessarily even. Note that although we define two different $J_{k}$ 's, it will always be clear from the context which one is meant. We denote by $Q_{k}$ the $k \times k$ matrix with ones on the south-west north-east diagonal and zeros elsewhere. Then the following characterization of pairs $(A, H)$ goes back to Weierstrass and Kronecker (See [GLR, T] for a complete proof).

THEOREM 2.1. Let $H$ be an invertible hermitian $n \times n$ matrix (over $F$ ), and let $A \in F^{n \times n}$ be $H$-selfadjoint. Then there exists an invertible $S$ over $F$ such that $S^{-1} A S$ and $S^{*} H S$ have the form

$$
S^{-1} A S=J_{k_{1}}\left(\lambda_{1}\right) \oplus \cdots \oplus J_{k_{\alpha}}\left(\lambda_{\alpha}\right) \oplus\left[J_{k_{\alpha+1}}\left(\lambda_{\alpha+1}\right) \oplus J_{k_{\alpha+1}}\left(\bar{\lambda}_{\alpha+1}\right)\right] \oplus \cdots \oplus\left[J_{k_{\beta}}\left(\lambda_{\beta}\right) \oplus J_{k_{\beta}}\left(\bar{\lambda}_{\beta}\right)\right]
$$

if $F=\mathrm{C}$, where $\lambda_{1}, \ldots, \lambda_{\alpha}$ are real and $\lambda_{\alpha+1}, \ldots, \lambda_{\beta}$ are non-real with positive imaginary parts;

$$
S^{-1} A S=J_{k_{1}}\left(\lambda_{1}\right) \oplus \cdots \oplus J_{k_{\alpha}}\left(\lambda_{\alpha}\right) \oplus J_{2 k_{\alpha+1}}\left(\lambda_{\alpha+1} \pm i \mu_{\alpha+1}\right) \oplus \cdots \oplus J_{2 k_{\beta}}\left(\lambda_{\beta} \pm i \mu_{\beta}\right),
$$

if $F=\mathbf{R}$, where $\lambda_{1}, \ldots, \lambda_{\beta}$ are real and $\mu_{\alpha+1}, \ldots, \mu_{\beta}$ are positive;

$$
S^{*} H S=\epsilon_{1} Q_{k_{1}} \oplus \cdots \oplus \epsilon_{\alpha} Q_{k_{\alpha}} \oplus Q_{2 k_{\alpha+1}} \oplus \cdots \oplus Q_{2 k_{\beta}}
$$

for both cases $(F=\mathbf{R}$ or $F=\mathbf{C})$, where the signs $\epsilon_{1}, \ldots, \epsilon_{\alpha}$ are \pm 1 . For a given pair $(A, H)$, where $A$ is $H$-selfadjoint, the canonical form (2.1), (2.3) (for $F=\mathbf{C}$ ) or (2.2), (2.3) (for $F=\mathbf{R})$ is unique up to permutation of $H$-orthogonal components in (2.3), and the same simultaneous permutation of the corresponding blocks in (2.1) or (2.2), as the case may be. 
We now discuss the spectral properties of $X^{[*]} X$ valid for $H$-plus matrices $X$. The following result was proved in [BMRRR2].

THEOREM 2.2. The $n \times n$ matrix $X$ is a strict $H$-plus matrix if and only if $X^{[*]} X$ has the following properties:

(a) $\sigma\left(X^{[*]} X\right) \subset[0, \infty)$;

(b) There exists $\mu>0$ such that there are no Jordan blocks of order exceeding 1 corresponding to the eigenvalues of $X^{[*]} X$ different from $\mu$. The eigenvectors corresponding to the eigenvalues smaller than $\mu$ are negative; those corresponding to the eigenvalues larger than $\mu$ are positive;

(c) There do not exist Jordan blocks of order exceeding 2 corresponding to the eigenvalue $\mu$ of $X^{[*]} X$; the blocks of length 2 have the positive sign in the canonical form of $\left(X^{[*]} X, H\right)$.

The matrix $X$ is an $H$-plus matrix with $\mu(X)=0$ if and only if $X^{[*]} X$ fails to satisfy at least one of (a), (b) and (c), and has, in addition, the following properties:

(d) $\sigma\left(X^{[*]} X\right) \subset \mathbf{R} ;$ and $0 \in \sigma\left(X^{[*]} X\right)$;

(e) There are no Jordan blocks of order exceeding 1 corresponding to the eigenvalues of $X^{[*]} X$ different from zero. The eigenvectors corresponding to the negative eigenvalues are negative; those corresponding to the positive eigenvalues are positive;

(f) There do not exist Jordan blocks of order exceeding 2 corresponding to the zero eigenvalue of $X^{[*]} X$; the blocks of size 2 have the positive sign in the canonical form of $\left(X^{[*]} X, H\right)$.

Other characterizations of strict $H$-plus matrices in the complex case can be obtained using [BG1]; in the terminology of [BG1] G-bounded means the same thing as strictly $(-G)$ plus in our terminology. Various characterizations of $(-H)$-bounded matrices can then be restated in our terminology as characterizations of strict $H$-plus matrices.

The description of other classes of $H$-plus matrices depends largely on the properties of $\mu(X)$ as an eigenvalue of $X^{[*]} X$. In this connection we note the following proposition.

PROPOSITION 2.3. Let $X$ be an $H$-plus matrix. Then $\mu(X)$ coincides with the minimal eigenvalue of $X^{[*]} X$ for which there exists an eigenvector $v$ such that $[v, v] \geq 0$. Moreover,

$$
\mu(X)=\min _{[u, u]=1}\left[X^{[*]} X u, u\right]
$$

if and only if there exists a positive vector $u$ such that $X^{[*]} X u=\mu(X) u$.

Observe that the set of $u \in F^{n}$ such that $[u, u]=1$ is not compact (if $H$ is indefinite), therefore, (2.4) is not guaranteed in general; see (1.1).

Proof. The first part is Corollary 3.7 in [BMRRR2]. We prove the second part. If there exists a positive vector $u$ such that $X^{[*]} X u=\mu(X) u$, then the minimum in (2.4) is assumed for a suitable scalar multiple of $u$. 
Conversely, assume there does not exist a positive vector $u$ such that $X^{[*]} X u=\mu(X) u$. Because of Theorem 2.2 and the first part of Proposition 2.3, $\mu(X)$ is an eigenvalue of $X^{[*]} X$ and $R_{\mu(X)}\left(X^{[*]} X\right)$ consists only of vectors in Jordan chains of length 2 and negative eigenvectors; all Jordan blocks of length 2 have the positive sign in the canonical form of $\left(X^{[*]} X, H\right)$ and there is at least one such block. Now let $\left\{u_{1}, v_{01}, \cdots, u_{r}, v_{0 r}, w_{1}, \cdots, w_{s}\right\}$ be a basis of $R_{\mu(X)}\left(X^{[*]} X\right)$ such that

$$
\left\{\begin{array}{l}
X^{[*]} X w_{i}=\mu(X) w_{i}, \quad\left[w_{i}, w_{j}\right]=0(i \neq j), \quad\left[w_{i}, w_{i}\right]=-1 \\
X^{[*]} X v_{0 i}=\mu(X) v_{0 i}+u_{i}, X^{[*]} X u_{i}=\mu(X) u_{i},\left[u_{i}, u_{i}\right]=0,\left[u_{i}, v_{0 i}\right]=1, \quad\left[v_{0 i}, v_{0 i}\right]=0 \\
{\left[u_{i}, u_{j}\right]=\left[u_{i}, v_{0 j}\right]=\left[v_{0 i}, v_{0 j}\right]=0(i \neq j), \quad\left[u_{i}, w_{j}\right]=\left[v_{0 i}, w_{j}\right]=0 .}
\end{array}\right.
$$
write

Assume that for some vector $x$ we have $[x, x]=1$ and $\left[X^{[*]} X x, x\right]=\mu(X)[x, x]$. We can

$$
x=\sum_{i=1}^{r}\left(\alpha_{i} v_{0 i}+\beta_{i} u_{i}\right)+\sum_{j=1}^{s} \gamma_{j} w_{j}+\sum_{k=1}^{t} \tilde{u}_{k}
$$

for some $\alpha$ 's, $\beta$ 's, $\gamma^{\prime}$ 's and $\tilde{u}$ 's, where $X^{[*]} X \tilde{u}_{k}=\lambda_{k} \tilde{u}_{k}$ with $\lambda_{k} \in[0,+\infty) \backslash\{\mu(X)\}$. Then

$$
[x, x]=\sum_{i=1}^{\pi} 2 \operatorname{Re} \alpha_{i} \bar{\beta}_{i}-\sum_{j=1}^{s}\left|\gamma_{j}\right|^{2}+\sum_{k=1}^{t}\left[\tilde{u}_{k}, \tilde{u}_{k}\right]
$$

and

$$
\left[X^{[*]} X x, x\right]=\sum_{i=1}^{r}\left(\left|\alpha_{i}\right|^{2}+2 \mu(X) \operatorname{Re} \alpha_{i} \bar{\beta}_{i}\right)-\mu(X) \sum_{j=1}^{s}\left|\gamma_{j}\right|^{2}+\sum_{k=1}^{t} \lambda_{k}\left[\tilde{u}_{k}, \tilde{u}_{k}\right],
$$

which imply

$$
0=\left[X^{[*]} X x, x\right]-\mu(X)[x, x]=\sum_{i=1}^{\tau}\left|\alpha_{i}\right|^{2}+\sum_{k=1}^{t}\left(\lambda_{k}-\mu(X)\right)\left[\tilde{u}_{k}, \tilde{u}_{k}\right],
$$

where the terms in the two sums are nonnegative. As a result, $\alpha_{1}=\cdots=\alpha_{r}=0$ and $\tilde{u}_{1}=\cdots=\tilde{u}_{t}=0$. Hence $x=\sum_{i=1}^{r} \beta_{i} u_{i}+\sum_{j=1}^{s} \gamma_{j} w_{j}$, which has the property $[x, x]=$ $-\sum_{j=1}^{s}\left|\gamma_{j}\right|^{2}$ and hence cannot satisfy $[x, x]=1$. Contradiction. Thus the mimimum in (1.1) is not assumed for any $x \in F^{n}$ with $[x, x]=1$.

If (2.4) holds instead of the weaker property (1.1), we say that $\mu(X)$ is attained. A sufficient (but generally not necessary) condition for attainability of $\mu(X)$ is that $X^{[*]} X$ be diagonalizable. However, if $H$ has only one positive eigenvalue, then $\mu(X)$ is attained if and only if $X^{[*]} X$ is diagonalizable.

The following connection between the canonical forms of $\left(X^{[*]} X, H\right)$ and $\left(X X^{[*]}, H\right)$ will be useful for characterizing the class $\mathcal{D P}$ and proving that $\mathcal{S P} \subseteq \mathcal{D P}$.

PROPOSITION 2.4. (a) The pairs $\left(X^{[*]} X, H\right)$ and $\left(X X^{[*]}, H\right)$ have the same canonical form for the positive eigenvalues of $X^{[*]} X$ (which are also the positive eigenvalues of $\left.X X^{[*]}\right)$, and they have opposite canonical form, $i . e$, the canonical form with the opposite signs, for the negative eigenvalues of $X^{[*]} X$ (which are also the negative eigenvalues of $X X^{[*]}$. 
(b) Assume that the real eigenvalues (if any) of $X^{[*]} X$ are nonnegative and the Jordan blocks of $X^{[*]} X$ corresponding to the zero eigenvalue (if $0 \in \sigma\left(X^{[*]} X\right)$ ) are all of size 1 and have the same sign in the canonical form of $\left(X^{[*]} X, H\right)$. Then $\left(X^{[*]} X, H\right)$ and $\left(X X^{[*]}, H\right)$ have the same canonical form.

Proof. Let us start from the fact that if $A$ and $B$ are $n \times n$ matrices such that $A B$ is either invertible or has Jordan blocks of order 1 only corresponding to the zero eigenvalue, and $\operatorname{rank}(A B)=\operatorname{rank}(B A)$, then $A B$ and $B A$ are similar. This follows from the general description of the relationship between the Jordan form of $A B$ and the Jordan form of $B A$ (see $[\mathrm{F}]$ ).

Let

$$
x_{0}, x_{1}, \ldots, x_{p}
$$

be a Jordan chain of $X^{[*]} X$ corresponding to a non-zero eigenvalue $\lambda$ :

$$
\begin{cases}\left(X^{[*]} X-\lambda I\right) x_{j}=x_{j-1} ; & j=1, \ldots, p ; \\ \left(X^{[*]} X-\lambda I\right) x_{0}=0, & x_{0} \neq 0 .\end{cases}
$$

Premultiplying these equalities by $X$ we see that $X x_{0} \neq 0$ and

$$
X x_{0}, X x_{1}, \ldots, X x_{p}
$$

is a Jordan chain of $X X^{[*]}$ corresponding to $\lambda$. Now

$$
\left[X x_{0}, X x_{p}\right]=\left[X^{[*]} X x_{0}, x_{p}\right]=\lambda\left[x_{0}, x_{p}\right] .
$$

This shows that the signs in the canonical forms of $\left(X^{[*]} X, H\right)$ and $\left(X X^{[*]}, H\right)$ corresponding to (2.5) and (2.6), respectively, are the same if $\lambda>0$ and are opposite if $\lambda<0$.

For part (b), assume, for example, that all signs of the Jordan blocks of $X^{[*]} X$ corresponding to the zero eigenvalue are +1 ; let $k$ be the number of such blocks. The number of positive eigenvalues of $H$ is equal to $k+\#$ positive eigenvalues of $\left.H\right|_{\mathcal{M}}$ \}, where $\mathcal{M}$ is the direct sum of root subspaces of $X^{[*]} X$ corresponding to non-zero eigenvalues. Observe that $\operatorname{dim} \mathcal{M}=n-k$. By part (a) of this proposition we have

$$
\#\left\{\text { positive eigenvalues of }\left.H\right|_{\mathcal{M}}\right\}=\#\left\{\text { positive eigenvalues of }\left.H\right|_{\mathcal{N}}\right\} \text {, }
$$

where $\mathcal{N}$ is the subspace defined analogously to $\mathcal{M}$, but using $X X^{[*]}$ in place of $X^{[*]} X$. By $[\mathrm{F}]$ we have $\operatorname{dim} \mathcal{M}=\operatorname{dim} \mathcal{N}$. Letting $\mathcal{N}_{0}=\operatorname{Ker}\left(X X^{[*]}\right)^{n}$, we must have that $\#\left\{\right.$ positive eigenvalues of $\left.\left.H\right|_{\mathcal{N}_{0}}\right\}=k$, but also $\operatorname{dim} \mathcal{N}_{0}=k$. In view of the canonical form of $\left(X X^{[*]}, H\right)$ it follows that the Jordan blocks of $X X^{[*]}$ corresponding to the zero eigenvalue are all of size 1 with the sign +1 in the canonical form. Now (b) follows form (a).

The second part of Proposition 2.4 can be easily restated without using the canonical form, as follows: Assume that $X^{[*]} X$ has no (real) negative eigenvalues and that $[u, u] \neq 0$ for every nonzero $u \in \operatorname{Ker} X^{[*]} X$ (if such $u$ exist). Then there exists an $H$-unitary $U$ such that $U^{-1} X^{[*]} X U=X X^{[*]}$.

For a description of the structure of doubly $H$-plus matrices we have to apply the result of Theorem 2.2 with $X$ replaced by $X^{[*]}$, and take into account Proposition 2.4.

THEOREM 2.5. An $n \times n$ matrix $X$ is doubly $H$-plus if and only if it is either a strict $H$-plus matrix or a non-strict $H$-plus matrix with the following additional properties: 
(i) $\sigma\left(X^{[*]} X\right)$ is nonnegative,

(ii) condition (f) of Theorem 2.2 applies to $X X^{[*]}$ (as well as to $X^{[*]} X$ ).

Strongly strict $H$-plus matrices are described in terms of $X^{[*]} X$ as follows.

THEOREM 2.6. The following statements are equivalent for an $n \times n$ matrix $X$ :

(i) $X \in \mathcal{S S P}$;

(ii) $X \in \mathcal{S P}, X^{[*]} X$ is diagonalizable, and the eigenvectors of $X^{[*]} X$ corresponding to the eigenvalue $\mu(X)$ are positive;

(iii) $\sigma\left(X^{[*]} X\right) \subset[0, \infty)$, for every eigenvector $u$ of $X^{[*]} X$ the inequality $[u, u] \neq 0$ holds, and if $u_{1}, u_{2}$ are eigenvectors of $X^{[*]} X$ corresponding to the eigenvalues $\lambda_{1}<\lambda_{2}$, respectively, then $\left[u_{1}, u_{1}\right]>0 \Rightarrow\left[u_{2}, u_{2}\right]>0$.

Proof. Suppose $X \in \mathcal{P}$, and $X^{[*]} X$ is not diagonalizable or $X^{[*]} X$ is diagonalizable but has a real eigenvalue $\mu$ of indefinite type. Then there exists $u \neq 0$ such that $[u, u]=0$ and $X^{[*]} X u=\mu u$. As a result, $[X u, X u]=0$ and hence $X \notin \mathcal{S S P}$.

Let $X \in \mathcal{S S P}$. Then $X^{[*]} X$ is diagonalizable and the eigenspace corresponding to the eigenvalue $\mu(X)$ is positive (cf. first part of Proposition 2.3 and the observation in the preceding paragraph). In fact, $\mu(X)>0$, since for $\mu(X)=0$ and $u$ a (positive) eigenvector of $X^{[*]} X$ corresponding to the zero eigenvector we would have $[X u, X u]=0$, which would imply $X \notin \mathcal{S S P}$. Conversely, assume that $X \in \mathcal{S P}, X^{[*]} X$ is diagonalizable, and $[u, u]>0$ for every $u \neq 0$ such that $X^{[*]} X u=\mu(X) u$. Consider a basic set of $H$-orthogonal eigenvectors $u_{1}, \cdots, u_{n}$ and corresponding (real) eigenvalues $\lambda_{1}, \cdots, \lambda_{n}$, where $u_{j}$ is positive (negative) if $\lambda_{j} \geq \mu(X)\left(\lambda_{j}<\mu(X)\right)$. Then for $u=u_{1}+\cdots+u_{n}$ we have

$$
\begin{aligned}
{[X u, X u] } & =\left[X^{[*]} X u, u\right]=\sum_{j=1}^{n}\left[X^{[*]} X u_{j}, u_{j}\right]=\sum_{j=1}^{n} \lambda_{j}\left[u_{j}, u_{j}\right] \\
& \geq \mu(X) \sum_{j=1}^{n}\left[u_{j}, u_{j}\right]=\mu(X)[u, u]
\end{aligned}
$$

Now if one of the eigenvalues $\lambda_{j}$ differs from $\mu(X)$ and the corresponding $u_{j}$ is nontrivial, then $\lambda_{j}\left[u_{j}, u_{j}\right]>\mu(X)\left[u_{j}, u_{j}\right]$ and hence $[X u, X u]>0$ whenever $[u, u] \geq 0$. Further, if $X^{[*]} X u=\mu(X) u$ and $u \neq 0$, then $[X u, X u]=\mu(X)[u, u]>0$. Consequently, if $[u, u] \geq 0$ and $u \neq 0$, we find $[X u, X u]>0$ and hence $X \in \mathcal{S S P}$. This proves the equivalence of (i) and (ii) in the theorem.

From the canonical form of the pair $\left(X^{[*]} X, H\right)$ we derive the equivalence of (ii) and (iii), taking into account the description of the class $\mathcal{S P}$ given in Theorem 2.2 and the characterization of $\mu(X)$ as the minimal eigenvalue of $X^{[*]} X$ with a nonnegative eigenvector (Proposition 2.3).

Combining Theorems 2.2 and 2.6 with Proposition 2.4 it follows that $X \in \mathcal{S S P}$ (resp. $X \in \mathcal{S P}$ ) if and only if $X^{[*]} \in \mathcal{S S P}$ (resp. $X^{[*]} \in \mathcal{S P}$ ). 
The following known inclusion relations follow immediately from Theorems 2.2, 2.5, 2.6:

$$
\mathcal{S S P} \subseteq \mathcal{S P} \subseteq \mathcal{D P} \subseteq \mathcal{P}
$$

The inclusion $\mathcal{S P} \subseteq \mathcal{D P}$ is well-known [Bo, P2]. It can also be derived using that $\mathcal{S P}=\{X$ : $X$ is $(-H)$-bounded $\}$, since $(-H)$-boundedness is preserved under the operation $X \mapsto X^{[*]}$ (see [BG1]). The inclusion $\mathcal{S S P} \subseteq \mathcal{S P}$ is observed in [IKL], Lemma 6.2. The following examples show that the inclusions in (2.7) are strict.

Example 2.1. $\mathcal{S P} \neq \mathcal{D P}$. Let

$$
X=\left[\begin{array}{lll}
0 & 1 & 0 \\
0 & 0 & 1 \\
0 & 0 & 0
\end{array}\right], \quad H=\left[\begin{array}{lll}
0 & 0 & 1 \\
0 & 1 & 0 \\
1 & 0 & 0
\end{array}\right] .
$$

Then using $X=X^{[*]}$ and $\left[X^{[*]} X u, u\right]=\left|u_{3}\right|^{2}$ for every $u=\left[\begin{array}{lll}u_{1} & u_{2} & u_{3}\end{array}\right]^{T} \in F^{3}$, we see that $X \in \mathcal{D} \mathcal{P} \backslash \mathcal{S P}$.

Example 2.2. $\mathcal{D} \mathcal{P} \neq \mathcal{P}$. Let

$$
X=\left[\begin{array}{ll}
0 & 1 \\
0 & 0
\end{array}\right], \quad H=\left[\begin{array}{cc}
1 & 0 \\
0 & -1
\end{array}\right] .
$$

Then $X^{[*]} X=\operatorname{diag}(0,-1)$ and $X X^{[*]}=\operatorname{diag}(-1,0)$. Hence $X \in \mathcal{P} \backslash \mathcal{D} \mathcal{P}$.

Example 2.3. To show that $\mathcal{D P}$ is strictly smaller than the set of $H$-plus matrices with spectrum in $[0, \infty)$, let

$$
H=\left[\begin{array}{llll}
0 & 0 & 0 & 1 \\
0 & 0 & 1 & 0 \\
0 & 1 & 0 & 0 \\
1 & 0 & 0 & 0
\end{array}\right], \quad X=\left[\begin{array}{llll}
0 & 0 & 0 & 0 \\
0 & 0 & 0 & 0 \\
a & b & c & d \\
e & f & g & h
\end{array}\right]
$$

where $a, \ldots, h$ are real. One easily computes that $X^{[*]} X=0$, so $X$ is an $H$-plus matrix. Furthermore, if we put $x=\left[\begin{array}{llll}a & b & c & d\end{array}\right]^{T}$ and $y=\left[\begin{array}{lll}e . f & g & h\end{array}\right]^{T}$, then

$$
X X^{[*]}=\left[\begin{array}{cccc}
0 & 0 & 0 & 0 \\
0 & 0 & 0 & 0 \\
\langle x, H y\rangle & \langle x, H x\rangle & 0 & 0 \\
\langle y, H y\rangle & \langle y, H x\rangle & 0 & 0
\end{array}\right]
$$

We can choose $x$ and $y$ so that $\langle x, H y\rangle=0,\langle x, H x\rangle=-1$, and $\langle y, H y\rangle=1$, for instance, take $x=2^{-1 / 2}\left[\begin{array}{cccc}1 & 0 & 0 & -1\end{array}\right]^{T}$ and $y=2^{-1 / 2}\left[\begin{array}{llll}0 & 1 & 1 & 0\end{array}\right]^{T}$. Then

$$
X X^{[*]}=\left[\begin{array}{cccc}
0 & 0 & 0 & 0 \\
0 & 0 & 0 & 0 \\
0 & -1 & 0 & 0 \\
1 & 0 & 0 & 0
\end{array}\right]
$$


So $X X^{[*]} e_{1}=e_{4}$, and $X X^{[*]} e_{4}=0$, while $X X^{[*]} e_{2}=-e_{3}$ and $X X^{[*]} e_{3}=0$. Moreover, $\left\langle H e_{1}, e_{4}\right\rangle=1$, and $\left\langle H e_{2},-e_{3}\right\rangle=-1$. Thus the canonical form of the pair $\left(X X^{[*]}, H\right)$ is

$$
\left(\left[\begin{array}{llll}
0 & 1 & 0 & 0 \\
0 & 0 & 0 & 0 \\
0 & 0 & 0 & 1 \\
0 & 0 & 0 & 0
\end{array}\right],\left[\begin{array}{rrrr}
0 & 1 & 0 & 0 \\
1 & 0 & 0 & 0 \\
0 & 0 & 0 & -1 \\
0 & 0 & -1 & 0
\end{array}\right]\right)
$$

so that $X^{[*]}$ is not an $H$-plus matrix (by Theorem 2.2).

Example 2.4. For complex numbers $a, b$, let $H=\left[\begin{array}{ll}0 & 1 \\ 1 & 0\end{array}\right], X=\left[\begin{array}{ll}0 & 0 \\ a & b\end{array}\right]$. Then $X^{[*]}=\left[\begin{array}{ll}\bar{b} & 0 \\ \bar{a} & 0\end{array}\right]$. One verifies that $X$ is always $H$-plus (as its image is an $H$-nonnegative subspace), but $X^{[*]}$ is $H$-plus if and only if $\operatorname{Re}(a \bar{b}) \geq 0$. Thus $X$ is doubly $H$-plus if and only if $\operatorname{Re}(a \bar{b}) \geq 0$. Note that in this example rank $X^{[*]} X \neq \operatorname{rank} X X^{[*]}$ (unless $\operatorname{Re}(a \bar{b})=0$.

Example 2.5. Let

$$
H=\left[\begin{array}{lll}
0 & 0 & 1 \\
0 & 1 & 0 \\
1 & 0 & 0
\end{array}\right], \quad X=\left[\begin{array}{lll}
a & b & c \\
0 & d & e \\
0 & 0 & f
\end{array}\right]
$$

where $a, b, c, d, e, f$ are complex numbers. A calculation shows that

$$
X^{[*]}=\left[\begin{array}{ccc}
\bar{f} & \bar{e} & \bar{c} \\
0 & \bar{d} & \bar{b} \\
0 & 0 & \bar{a}
\end{array}\right], \quad X^{[*]} X=\left[\begin{array}{ccc}
\bar{f} a & \bar{f} b+\bar{e} d & \bar{f} c+|e|^{2}+\bar{c} f \\
0 & |d|^{2} & \bar{d} e+\bar{b} f \\
0 & 0 & f \bar{a}
\end{array}\right] .
$$

Now Theorem 2.2 shows that $X \in \mathcal{S P}$ if and only if one of the following two sets of equations. and inequalities is satisfied:

$$
\begin{gathered}
0<\bar{f} a<|d|^{2}, \quad|\bar{f} b+\bar{e} d|^{2} \leq\left(|d|^{2}-f \bar{a}\right)\left(\bar{f} c+|e|^{2}+\bar{c} f\right), \\
0<\bar{f} a=|d|^{2}, \quad \bar{f} b+\bar{e} d=0, \quad \bar{f} c+|e|^{2}+\bar{c} f \geq 0 .
\end{gathered}
$$

Theorem 2.6 shows that $X$ is never in $\mathcal{S S P}$. Thus, $\mathcal{S S P} \neq \mathcal{S P}$.

Theorem 2.5 shows that if $X \in \mathcal{P}$ then $X \in \mathcal{D P} \backslash \mathcal{S P}$ if and only if

(a) $\sigma\left(X^{[*]} X\right) \subset[0, \infty)$, and $0 \in \sigma\left(X^{[*]} X\right)$,

(b) $X^{[*]} X$ has only $H$-positive eigenvectors corresponding to its strictly positive eigenvalues (if any),

(c) $X^{[*]} X$ has Jordan blocks of order at most two with the zero eigenvalue, and all signs in the canonical form of $\left(X^{[*]} X, H\right)$ corresponding to the Jordan blocks of order two with the zero eigenvalue are +1 , 
(d) $X X^{[*]}$ has Jordan blocks of order at most two with the zero eigenvalue, and all signs in the canonical form of $\left(X X^{[*]}, H\right)$ corresponding to Jordan blocks of order two with zero eigenvalue are all +1 .

One might think that the following would hold: if $X \in \mathcal{P}$ then $X \in \mathcal{D P} \backslash \mathcal{S P}$ if and only if (a), (b), (c) above hold and only the first part of (d) is required, i.e., only the fact that $X X^{[*]}$ has Jordan blocks of order at most two with zero eigenvalue. Example 2.3 shows that this conjecture fails.

If $X \in \mathcal{P} \backslash \mathcal{S P}$ then in general condition (d) above will not hold, but we can say the following about the canonical form of $\left(X X^{[*]}, H\right)$ :

(i) Corresponding to the zero eigenvalue the total algebraic multiplicities of $X^{[*]} X$ and $X X^{[*]}$ are the same (this follows from $[\mathrm{F}]$ as well as from the fact that this is obviously true for all nonzero eigenvalues of $X^{[*]} X$ and $X X^{[*]}$ ).

(ii) The Jordan blocks of $X X^{[*]}$ with zero eigenvalue have order at most 3 (this follows from $[\mathrm{F}]$ as well as from the identity $\left.\left(X X^{[*]}\right)^{3}=X\left(X^{[*]} X\right)^{2} X^{[*]}\right)$.

(iii) The signs in the canonical form of $\left(X X^{[*]}, H\right)$ corresponding to Jordan blocks of order three with zero eigenvalue are all +1 .

To prove part (iii), let $x_{1} \neq 0, x_{2}, x_{3}$ be a Jordan chain of length three for $X X^{[*]}$, i.e., $X X^{[*]} x_{1}=0, X X^{[*]} x_{2}=x_{1}$, and $X X^{[*]} x_{3}=x_{2}$. Then we have $X^{[*]} X X^{[*]} x_{1}=0, X^{[*]} X X^{[*]} x_{2}$ $=X^{[*]} x_{1}$, and $X^{[*]} X X^{[*]} x_{3}=X^{[*]} x_{2}$. As $X^{[*]} X$ has Jordan blocks of order two at most corresponding to the zero eigenvalue, it follows that $X^{[*]} x_{1}=0$, and $X^{[*]} x_{2}, X^{[*]} x_{3}$ form a Jordan chain of length two as $X^{[*]} x_{2} \neq 0$. The sign in the canonical form of $\left(X X^{[*]}, H\right)$ corresponding to this Jordan chain of length three is given by the sign of $\left\langle H x_{2}, x_{2}\right\rangle=\left\langle H X X^{[*]} x_{3}, x_{2}\right\rangle=$ $\left\langle H X^{[*]} x_{3}, X^{[*]} x_{2}\right\rangle$. As the signs in the canonical form of $\left(X^{[*]} X, H\right)$ corresponding to Jordan blocks of order two with zero eigenvalue is +1 , we see that $\left\langle H X^{[*]} x_{3}, X^{[*]} x_{2}\right\rangle=1$. This proves (iii).

Observe, by the way, that the above argument can be applied (with obvious modifications) to prove the following. Let $k$ be the largest size of a Jordan block corresponding to the zero eigenvalue in the canonical form of $\left(X^{[*]} X, H\right)$, and let there be $p$ signs +1 and $q$ signs -1 in the canonical form of the blocks of size $k$ corresponding to the zero eigenvalue. Then the number of signs +1 in the canonical form of the pair $\left(X X^{[*]}, H\right)$ corresponding to the blocks of size $k+1$ with zero eigenvalue does not exceed $p$, and the number of signs -1 corresponding to the blocks of size $k+1$ does not exceed $q$.

To study the topological structure of the classes $\mathcal{S P}, \mathcal{D P}$ and $\mathcal{P}$ in the next section, we introduce some additional sets of $H$-plus matrices. Given an $H$-plus matrix $X$, let $\mathcal{M}(X)$ be the set of all nonnegative numbers $\mu$ such that

$$
\left[X^{[*]} X z, z\right] \geq \mu[z, z], \quad z \in F^{n} .
$$

Clearly, $\mathcal{M}(X)$ is non-empty, because $\mu(X) \in \mathcal{M}(X)$. In fact, $\mathcal{M}(X)$ is a closed interval, possibly consisting of one point only. Define

$$
\begin{aligned}
& \mathcal{S P} \mathcal{P}_{u}=\{X \in \mathcal{S P}: \mathcal{M}(X)=\{\mu(X)\}\} \\
& \mathcal{D P} \mathcal{P}_{u}=\{X \in \mathcal{D P}: \mathcal{M}(X)=\{\mu(X)\}\}
\end{aligned}
$$




$$
\mathcal{P}_{u}=\{X \in \mathcal{P}: \mathcal{M}(X)=\{\mu(X)\}\} .
$$

The classes $\mathcal{S} \mathcal{P}_{u}, \mathcal{D P} \mathcal{P}_{u}$ and $\mathcal{P}_{u}$ are distinct and differ from the respective classes $\mathcal{S P}$, $\mathcal{D P}$ and $\mathcal{P}$. The next theorem gives a complete description of the classes $\mathcal{S} \mathcal{P}_{u}, \mathcal{D} \mathcal{P}_{u}$ and $\mathcal{P}_{u}$. Moreover, we prove that $\mathcal{S} \mathcal{S P}=\mathcal{S P} \backslash \mathcal{S P}$.

THEOREM 2.7. We have

$$
\begin{aligned}
\mathcal{S P} \mathcal{P}_{u} & =\left\{X \in \mathcal{S P}: R_{\mu(X)}\left(X^{[*]} X\right) \text { is indefinite }\right\} \\
\mathcal{S P} \backslash \mathcal{S P} \mathcal{P}_{u} & =\left\{X \in \mathcal{S P}: R_{\mu(X)}\left(X^{[*]} X\right) \text { is positive }\right\}=\mathcal{S S P} \\
\mathcal{D P} \mathcal{P}_{u} & =(\mathcal{D P} \backslash \mathcal{S P}) \cup \mathcal{S P} \mathcal{P}_{u} \\
\mathcal{P}_{u} & =(\mathcal{P} \backslash \mathcal{S P}) \cup \mathcal{S P} \mathcal{P}_{u} .
\end{aligned}
$$

Proof. Because $\mu(X)$ is the infimum of $[X u, X u]$ over all $u$ with $[u, u]=1$, one sees immediately that $\mu(X)$ is the maximum of $\mathcal{M}(X)$. So, if $\mu(X)=0$ then $\mathcal{M}(X)=\{\mu(X)\}=$ $\{0\}$. This proves the part of the theorem concerning $\mathcal{D} \mathcal{P}_{u}$ and $\mathcal{P}_{u}$.

It remains to prove the equality

$$
\mathcal{S P} \backslash \mathcal{S} \mathcal{P}_{u}=\left\{X \in \mathcal{S P}: R_{\mu(X)}\left(X^{[*]} X\right) \text { is positive }\right\}
$$

Suppose $X$ is in $\mathcal{S P} \backslash \mathcal{S P} \mathcal{P}_{u}$, and let $\mu_{1}<\mu(X)$ be another number in $\mathcal{M}(X)$. Assume first that $X^{[*]} X$ has a block of order two corresponding to $\mu(X)$. Consider a vector $x$ which is constructed as follows. Let $S$ be a matrix that transforms the pair $\left(X^{[*]} X, H\right)$ to canonical form, and let $y$ be a vector which has zeros everywhere, except in the two positions corresponding to a Jordan block of order two for $X^{[*]} X$, where it has $(1, \alpha)^{T}$. (For simplicity, we take $\alpha$ real.) Then we take $x=S^{-1} y$. We obtain

$$
[X x, X x]=\left[X^{[*]} X x, x\right]=\left\langle\left[\begin{array}{ll}
0 & 1 \\
1 & 0
\end{array}\right]\left[\begin{array}{cc}
\mu(X) & 1 \\
0 & \mu(X)
\end{array}\right]\left[\begin{array}{l}
1 \\
\alpha
\end{array}\right],\left[\begin{array}{l}
1 \\
\alpha
\end{array}\right]\right\rangle=\alpha^{2}+2 \mu(X) \alpha .
$$

But $[X x, X x] \geq \mu_{1}[x, x]=2 \mu_{1} \alpha$. So, we get that for all real $\alpha$ the following should hold: $\alpha^{2}+2 \mu(X) \alpha \geq 2 \mu_{1} \alpha$. However, it is clearly possible to choose $\alpha$ such that this fails. This shows that $X^{[*]} X$ cannot have a Jordan block of size two corresponding to $\mu(X)$.

Next, assume that $X^{[*]} X$ has two Jordan blocks of size one with opposite signs in the canonical form. Construct a vector $y$ in a similar way as in the previous paragraph, except that the non-zero entries now correspond to the two blocks of order one with opposite signs, and let again $x=S^{-1} y$. Then

$$
[X x, X x]=\left[X^{[*]} X x, x\right]=\left\langle\left[\begin{array}{cc}
1 & 0 \\
0 & -1
\end{array}\right]\left[\begin{array}{cc}
\mu(X) & 0 \\
0 & \mu(X)
\end{array}\right]\left[\begin{array}{l}
1 \\
\alpha
\end{array}\right],\left[\begin{array}{l}
1 \\
\alpha
\end{array}\right]\right\rangle=\mu(X)\left(1-\alpha^{2}\right) .
$$

As before, this should be larger than or equal to $\mu_{1}[x, x]=\mu_{1}\left(1-\alpha^{2}\right)$, and clearly it is possible to choose $\alpha$ such that this is violated. So, we have shown that if $X$ is in $\mathcal{S P} \backslash \mathcal{S P} \mathcal{P}_{u}$, then the generalized eigenspace of $X^{[*]} X$ corresponding to $\mu(X)$ is not indefinite. In view of Proposition 2.3 this shows that $R_{\mu(X)}\left(X^{[*]} X\right)$ is positive. 
Conversely, let $R_{\mu(X)}\left(X^{[*]} X\right)$ be positive. Then with respect to a suitable basis we have that

$$
X^{[*]} X=\left[\begin{array}{cc}
A_{1} & 0 \\
0 & A_{2}
\end{array}\right], \quad H=\left[\begin{array}{cc}
I & 0 \\
0 & -I
\end{array}\right],
$$

where $A_{1}$ is a diagonal matrix with diagonal entries that are larger than or equal to $\mu(X)$, and $A_{2}$ is a diagonal matrix with diagonal entries that are strictly smaller than $\mu(X)$. Let $\mu$ be any number smaller then $\mu(X)$ and larger than $\mu_{-}(X):=\max \sigma\left(A_{2}\right)$. Let $x$ be any vector in $\mathrm{C}^{n}$, and decompose $x$ as $x=\left[\begin{array}{ll}x_{1} & x_{2}\end{array}\right]^{T}$, corresponding to the decomposition of $X^{[*]} X$ above. Then

$$
[X x, X x]=\left\langle A_{1} x_{1}, x_{1}\right\rangle-\left\langle A_{2} x_{2}, x_{2}\right\rangle>\mu\left(\left\langle x_{1}, x_{1}\right\rangle-\left\langle x_{2}, x_{2}\right\rangle\right)=\mu[x, x] .
$$

Observe that this shows that $\mathcal{M}(X) \supseteq\left[\mu_{-}(X), \mu(X)\right]$. This proves the proposition.

All seven classes $\mathcal{S S P}, \mathcal{S P}, \mathcal{D} \mathcal{P}, \mathcal{P}, \mathcal{S P} \mathcal{P}_{u}, \mathcal{D} \mathcal{P}_{u}$ and $\mathcal{P}_{u}$ are invariant under $H$-unitary equivalence (two matrices $Y$ and $Z$ are called $H$-unitarily equivalent if $Y=U Z V$ for some $H$-unitary matrices $U$ and $V$ ). Remark also that $\mu(X)=\mu(Y)$ if $X$ and $Y$ are $H$-unitarily equivalent $H$-plus matrices. The verification of these facts is straightforward.

We conclude this section with some results on the multiplicative structure of the various classes of $H$-plus matrices. We start with a lemma closely related to Theorem 2.7 of [BG1].

LEMMA 2.8. If the matrices $X$ and $Y$ are in one of the sets $\mathcal{P}, \mathcal{S P}, \mathcal{D P}, \mathcal{S S P}$, then the product $X Y$ is in the same set. Moreover,

$$
\mu(X Y) \geq \mu(X) \mu(Y) .
$$

Proof. Let $X, Y \in \mathcal{P}$. Then for every $x \in F^{n}$ we have

$$
[X Y x, X Y x] \geq \mu(X)[Y x, Y x] \geq \mu(X) \mu(Y)[x, x] .
$$

This verifies (2.8) and proves Lemma 2.8 for the sets $\mathcal{P}$ and $\mathcal{S P}$. If $X, Y \in \mathcal{D P}$, then the inequality

$$
\mu\left((X Y)^{[*]}\right)=\mu\left(Y^{[*]} X^{[*]}\right) \geq \mu\left(Y^{[*]}\right) \mu\left(X^{[*]}\right)
$$

implies that $(X Y)^{[*]} \in \mathcal{P}$. Therefore, $X Y \in \mathcal{D} \mathcal{P}$, as required. Finally, to prove Lemma 2.8 for $\mathcal{S S P}$ observe that

$$
\mathcal{M}(X Y) \supseteq \mathcal{M}(X) \cdot \mathcal{M}(Y)
$$

which can be proved analogously to (2.9). Now apply the equality $\mathcal{S S P}=\mathcal{S P} \backslash \mathcal{S P}$ proved in Theorem 2.7. Incidentally, this proves that $X Y \in \mathcal{S S P}$ if both $X$ and $Y$ belong to $\mathcal{S P}$ and at least one of them belongs to $\mathcal{S S P}$.

Let us now characterize the subgroups of the semigroup $\mathcal{P}$. Consider the set

$$
\mathcal{G}=\{c U: c>0, U \text { is } H \text {-unitary }\} .
$$

Clearly, $\mathcal{G}$ is a multiplicative group contained in $\mathcal{P}$. It turns out that every subgroup of $\mathcal{P}$ is contained in $\mathcal{G}$. This follows from the description of invertible $H$-plus matrices having $H$-plus inverses (this description appears in [Bo], and in [LS] for the ice-cream cone).

THEOREM 2.9. The following three statements are equivalent: 
(a) $X$ is an invertible $n \times n$ matrix such that both $X$ and $X^{-1}$ are $H$-plus matrices.

(b) $X$ is an $H$-plus matrix and every $v \in F^{n}$ such that $[v, v] \geq 0$ can be written in the form $v=X u$ where $[u, u] \geq 0$.

(c) $X \in \mathcal{G}$.

Proof. $[(c) \Longrightarrow(a)]$ and $[(c) \Longrightarrow(b)]$ are obvious.

$[(a) \Longrightarrow(c)]$ Let $X$ be an invertible matrix such that $X, X^{-1} \in \mathcal{P}$. Since $\mu(X)$ is an eigenvalue of $X$, we must have that both $\mu(X)$ and $\mu\left(X^{-1}\right)$ are positive, i.e., $X$ and $X^{-1}$ are strict $H$-plus matrices. Apply Theorem 2.2 to the strict $H$-plus matrix $X$. Let $Y=X^{[*]} X$. It follows that there exists a positive number $\mu$ such that every eigenvalue $\lambda_{+}$of $Y$ which is larger than $\mu$ has an $H$-positive eigenspace, whereas every eigenvalue $\lambda_{-}$of $Y$ which is smaller than $\mu$ has an $H$-negative eigenspace. (Note that by Theorem 2.2 and invertibility of $Y$ all eigenvalues of $Y$ are positive). But then the eigenvalue $\lambda_{+}^{-1}$ of $Y^{-1}$ having an $H$ positive eigenspace is smaller than $\mu^{-1}$ which in turn is smaller than the eigenvalue $\lambda_{-}^{-1}$ of $Y^{-1}$ having an $H$-negative eigenspace. This contradicts Theorem 2.2 applied to the strict $H$-plus matrix $X_{0}=\left(X^{-1}\right)^{[*]}$ (note that $Y^{-1}=X_{0}^{[*]} X_{0}$ ), unless $\mu$ is the sole eigenvalue of $Y$. Now $\sigma(Y)=\{\mu\}$, and by Theorem 2.2 the Jordan blocks in the Jordan form of $Y$ may be of size 1 or 2 only, and the blocks of size 2 (if any) must have the positive sign in the canonical form of $(Y, H)$. The equality $\left[\begin{array}{cc}\mu & 1 \\ 0 & \mu\end{array}\right]^{-1}=\left[\begin{array}{cc}\mu^{-1} & -\mu^{-2} \\ 0 & \mu^{-1}\end{array}\right]$ shows that the Jordan blocks of size 2 in the Jordan form of $Y^{-1}$ have the negative sign in the canonical form of $\left(Y^{-1}, H\right)$. This again contradicts Theorem 2.2 applied to $X_{0}$, and we must conclude that there are no Jordan blocks of size 2 in the Jordan form of $Y$. In other words, $Y=\mu I$, and therefore $X \in \mathcal{G}$.

$[(b) \Longrightarrow(a)]$ Let $X \in \mathcal{P}$ have the property that every $v \in F^{n}$ such that $[v, v] \geq 0$ can be written in the form $v=X u$ where $[u, u] \geq 0$, and let

$$
\mathcal{K}=\left\{x \in F^{n}:[x, x] \geq 0\right\} .
$$

Then $X[\mathcal{K}]=\mathcal{K}$. Also $X[-\mathcal{K}]=-\mathcal{K}$ and $X[\mathcal{K}+(-\mathcal{K})]=\mathcal{K}+(-\mathcal{K})$. Since $F^{n}=\mathcal{K}+(-\mathcal{K})$, the matrix $X$ is invertible and $X^{-1} \in \mathcal{P}$.

\section{Interiors and Closures}

In this section we determine the closure and the interior of the three sets $\mathcal{S P}, \mathcal{D P}$ and $\mathcal{P}$. First, in Theorem 3.1 we discuss the closures of these classes of $H$-plus matrices. Theorems 3.2 and 3.3 are devoted to the interiors of these sets.

THEOREM 3.1. The sets $\mathcal{P}$ and $\mathcal{D} \mathcal{P}$ of $H$-plus and doubly $H$-plus matrices are closed.

Proof. Let $\left\{X_{m}\right\}_{m=1}^{\infty}$ be a sequence of $H$-plus matrices that converges to a matrix $X$. By (1.2) we have

$$
\left[X_{m}^{[*]} X_{m} z, z\right] \geq \mu\left(X_{m}\right)[z, z], \quad z \in F^{n} .
$$


Further, $\mu\left(X_{m}\right)$ is an eigenvalue of $X_{m}^{[*]} X_{m}$ (see Proposition 2.3). The sequence $\left\{X_{m}^{[*]} X_{m}\right\}_{m=1}^{\infty}$ is obviously bounded, therefore the sequence $\left\{\mu\left(X_{m}\right)\right\}_{m=1}^{\infty}$ is bounded as well. Passing to a subsequence of $\left\{X_{m}\right\}_{m=1}^{\infty}$, if necessary, we can assume that the sequence $\left\{\mu\left(X_{m}\right)\right\}_{m=1}^{\infty}$ converges to some $\mu \geq 0$. Now clearly the matrix

$$
H\left(X^{[*]} X-\mu I\right)
$$

is the limit of the sequence of positive semidefinite matrices

$$
H\left(X_{m}^{[*]} X_{m}-\mu\left(X_{m}\right) I\right), \quad m=1,2, \cdots,
$$

and therefore (3.1) is itself positive semidefinite. The positive semidefiniteness of (3.1) easily implies that $X$ is an $H$-plus matrix. In other words, $\mathcal{P}$ is closed.

The fact that $\mathcal{D P}$ is closed is now immediate from the identity

$$
\mathcal{D P}=\mathcal{P} \cap\left\{X^{[*]}: X \in \mathcal{P}\right\}
$$

which completes the proof.

In contrast, the set $\mathcal{S P}$ of strictly $H$-plus matrices is not closed. Indeed, take $X \in \mathcal{S P}$. Then $\delta X \in \mathcal{S P}$ for all $\delta>0$, but the zero matrix is not strictly $H$-plus.

The closure of $\mathcal{S P}$ is obviously contained in $\mathcal{D P}$. Our conjecture is that the closure of $\mathcal{S P}$ coincides with $\mathcal{D P}$. In Section 4 we will prove this for the case when $H$ has only one positive eigenvalue. For more general $H$, the problem is open.

THEOREM 3.2. The set $\mathcal{S S P}$ is open.

Proof. Let $X \in \mathcal{S S P}$. Using Theorem 2.6(iii) and a general result on perturbations of $H$-selfadjoint matrices (Theorem III.1.1 in [GLR]), it follows that there are a positive $\mu$ (in fact, one can choose any positive $\mu<\mu(X)$ ) and a positive $\epsilon$ such that if $\|Y-X\|<\epsilon$ then the spectrum of $Y^{[*]} Y$ is real with $\mu \notin \sigma\left(Y^{[*]} Y\right), Y^{[*]} Y$ is diagonalizable, and every eigenvector of $Y^{[*]} Y$ corresponding to an eigenvalue $\lambda>\mu$ (resp. $\lambda<\mu$ ) is positive (resp. negative). Fix such an $Y$. Write any nonzero vector $u$ in the form $u=u_{1}+\cdots+u_{m}$, where $u_{j}$ is an eigenvector of $Y^{[*]} Y$ corresponding to the eigenvalue $\lambda_{j}$, and the eigenvalues $\lambda_{1}, \cdots, \lambda_{m}$ are distinct. In particular, the vectors $u_{1}, \cdots, u_{m}$ are $H$-orthogonal. Now

$$
\left[Y^{[*]} Y u, u\right]=\sum_{j} \lambda_{j}\left[u_{j}, u_{j}\right]=\mu \sum_{j}\left[u_{j}, u_{j}\right]+\sum_{j}\left(\lambda_{j}-\mu\right)\left[u_{j}, u_{j}\right] \geq \mu[u, u] .
$$

It follows that $Y \in \mathcal{S P}$. By Theorem $2.2, Y^{[*]} Y$ cannot have negative eigenvalues, hence by Theorem 2.6(iii) in fact $Y \in \mathcal{S S P}$.

For the interiors we have the following result.

THEOREM 3.3. The interiors of each of the sets $\mathcal{S P}, \mathcal{D P}$ and $\mathcal{P}$ consist of precisely those elements of $\mathcal{S P}$ which do not belong to $\mathcal{S P}$.

Proof. In view of the equality $\mathcal{S P} \backslash \mathcal{S P} \mathcal{P}_{u}=\mathcal{S S P}$ (Theorem 2.7) and Theorem 3.2, we only have to show that every $X \in \mathcal{S} \mathcal{P}_{u}$ and every $X \in \mathcal{P} \backslash \mathcal{S P}$ can be approximated by matrices not belonging to $\mathcal{P}$. 
We start with $X \in \mathcal{S P}_{u}$, and assume that $\left(X^{[*]} X, H\right)$ is given in canonical form. Then the restriction of $X^{[*]} X$ to $R_{\mu(X)}\left(X^{[*]} X\right)$ has the following canonical form:

$$
\begin{aligned}
& \left(\bigoplus_{r}\left[\begin{array}{cc}
\mu(X) & 1 \\
0 & \mu(X)
\end{array}\right] \oplus \operatorname{diag}(\mu(X), \cdots, \mu(X), \mu(X), \cdots, \mu(X)),\right. \\
& \left.\bigoplus_{r}\left[\begin{array}{ll}
0 & 1 \\
1 & 0
\end{array}\right] \oplus \operatorname{diag}(1, \cdots, 1,-1, \ldots,-1)\right),
\end{aligned}
$$

where there is at least one Jordan block of length 2 or a negative and a positive eigenvectors corresponding to the eigenvalue $\mu(X)$ of $X^{[*]} X$ (in other words, if there are no Jordan blocks of length 2 , then $s \geq 1, t \geq 1$, where $s$ and $t$ are the number of 1 's and the number of -1 's in the diagonal matrix in (3.2), respectively). Now let $A$ be the $H$-selfadjoint matrix defined by the properties that $A u=\sqrt{\alpha} u$ for every eigenvector $u$ of $X^{[*]} X$ corresponding to an eigenvalue $\alpha \neq \mu(X)$, whereas the restriction of $A$ to the generalized eigenspace $R_{\mu(X)}\left(X^{[*]} X\right)$ has the form

$$
\bigoplus_{r}\left[\begin{array}{cc}
\sqrt{\mu(X)} & \frac{1}{2 \sqrt{\mu(X)}} \\
0 & \sqrt{\mu(X)}
\end{array}\right] \oplus \operatorname{diag}(\sqrt{\mu(X)}, \cdots, \sqrt{\mu(X)}) .
$$

Clearly, $A^{2}=X^{[*]} X$. Then there is an $H$-unitary matrix $U$ such that $X=U A$. In fact, $c A$ is the modulus (in the sense of Potapov [P2]) of $c X$, where the positive constant $c$ is chosen so that $c X$ is an $-H$-contraction, see [P2], Lemma 3.1 and Theorem 2.4 in [BMRRR2]. Fix $\epsilon, 0<\epsilon<\sqrt{\mu(X)}$, and define the matrix $A_{\epsilon}$ by letting

$$
\begin{aligned}
A_{\epsilon}= & \left(\bigoplus_{r}\left[\begin{array}{cc}
\sqrt{\mu(X)}+\epsilon & \frac{1}{2 \sqrt{\mu(X)}} \\
-\epsilon & \sqrt{\mu(X)}+\epsilon
\end{array}\right] \oplus\right. \\
& \oplus \operatorname{diag}(\sqrt{\mu(X)-\epsilon}, \cdots, \sqrt{\mu(X)-\epsilon}, \sqrt{\mu(X)+\epsilon}, \cdots, \sqrt{\mu(X)+\epsilon}))
\end{aligned}
$$

on the subspace $R_{\sqrt{\mu(X)}}(A)=R_{\mu(X)}\left(X^{[*]} X\right)$ and $A_{\epsilon}=A$ on all other generalized eigenspaces of $A$. Let

$$
X_{\epsilon}=U A_{\epsilon}
$$

We compute

$$
\begin{aligned}
\left.X_{\epsilon}^{[*]} X_{\epsilon}\right|_{R_{\mu(X)}\left(X^{[*]} X\right)} & =\bigoplus_{r}\left[\begin{array}{cc}
(\sqrt{\mu(X)}+\epsilon)^{2}-\frac{\epsilon}{2 \sqrt{\mu(X)}} & \frac{\sqrt{\mu(X)}+\epsilon}{\sqrt{\mu(X)}} \\
-2 \epsilon(\sqrt{\mu(X)}+\epsilon) & (\sqrt{\mu(X)}+\epsilon)^{2}-\frac{\epsilon}{2 \sqrt{\mu(X)}}
\end{array}\right] \\
& \oplus \operatorname{diag}(\mu(X)-\epsilon, \cdots, \mu(X)-\epsilon, \mu(X)+\epsilon, \cdots, \mu(X)+\epsilon) .
\end{aligned}
$$

If a $2 \times 2$ Jordan block is present in (3.2) this matrix has the complex eigenvalues $(\sqrt{\mu(X)}+$ $\epsilon)^{2}-(\epsilon / 2 \sqrt{\mu(X)})+i(\sqrt{\mu(X)}+\epsilon)(2 \epsilon / \sqrt{\mu(X)})^{1 / 2}$. If there is no $2 \times 2$ Jordan block in (3.2) 
this matrix has positive eigenvectors at the eigenvalue $\mu(X)-\epsilon$ and negative eigenvectors at the eigenvalue $\mu(X)+\epsilon$. Thus $X_{\epsilon} \notin \mathcal{P}$ for all $\epsilon \in(0, \sqrt{\mu(X)})$.

Now let $X \in \mathcal{P} \backslash \mathcal{S P}$. If $X \in \mathcal{P}$ has negative eigenvalues, then approximating $X$ by invertible matrices $X_{\epsilon}$, one sees that $X_{\epsilon}^{[*]} X_{\epsilon}$ has eigenvalues in the open left half-plane and is invertible for nonzero $\epsilon$ small enough. According to Theorem $2.2, X_{\epsilon} \notin \mathcal{P}$ for nonzero $\epsilon$ small enough.

Now let $X \in \mathcal{P} \backslash \mathcal{S P}$ have only nonnegative eigenvalues. Then $X$ is not invertible. Take $0 \neq y \in \mathbf{R}^{n}$ and take $v \in \operatorname{Ker} X$ with $\|v\|=1$. Put $X_{\epsilon}=X+\epsilon y v^{*}$. Then

$$
\begin{aligned}
{\left[X_{\epsilon} v, X_{\epsilon} v\right] } & =[X v, X v]+\epsilon\|v\|^{2}[y, X v]+\epsilon\|v\|^{2}[X v, y]+\epsilon^{2}\|v\|^{4}[y, y] \\
& =\epsilon^{2}[y, y] .
\end{aligned}
$$

Thus if $[v, v] \geq 0$, it suffices to choose $y$ with $[y, y]<0$ to prove that $X_{\epsilon} \notin \mathcal{P}$. It remains to consider the case where every nontrivial $v \in \operatorname{Ker} X$ satisfies $[v, v]<0$. By Proposition 2.3 there is a vector $v \in \operatorname{Ker} X^{[*]} X$ with $\|v\|=1$ and $[v, v] \geq 0$. Hence $v \notin \operatorname{Ker} X$, and therefore $[X v, y]<0$ for some vector $y$. Now take $X_{\epsilon}=X+\epsilon y v^{*}$. We obtain

$$
\left[X_{\epsilon} v, X_{\epsilon} v\right]=2 \epsilon[X v, y]+\epsilon^{2}[y, y] \text {. }
$$

Then for $\epsilon>0$ small enough we have $\left[X_{\epsilon} v, X_{\epsilon} v\right]<0$. Consequently, $X_{\epsilon} \notin \mathcal{P}$ for $\epsilon>0$ small enough, which completes the proof.

Although a description of the closure of $\mathcal{S P}$ remains an open problem in general, the perturbation used in the proof of Theorem 3.3 allows us to show that the sets $\mathcal{S P}$ and $\mathcal{S S P}$ have the same closure. Indeed, in view of Theorem 2.7 we have only to see that every $X \in \mathcal{S P} \mathcal{P}_{u}$ can be aproximated by matrices in $\mathcal{S S P}$. To this end, we use formula (3.3), where now $\epsilon$ is close to zero but negative. In the vicinity of $\mu(X)$, the matrix $X_{\epsilon}^{[*]} X_{\epsilon}$ has now only real eigenvalues $1 \pm \epsilon$ and

$$
\lambda_{ \pm}=(1+\epsilon)^{2}-\frac{\epsilon}{2} \pm(1+\epsilon) \sqrt{-2 \epsilon}
$$

(for every $2 \times 2$ Jordan block present in (3.2)); we assume for simplicity of the formulas that $\mu(X)=1$. A calculation shows that the eigenvectors of $X_{\epsilon}^{[*]} X_{\epsilon}$ corresponding to the eigenvalue $\lambda_{+}$(resp. $\lambda_{-}$) are positive (resp. negative). By Theorem $2.7, X_{\epsilon} \in \mathcal{S S P}$.

\section{Special Case: H Has One Positive Eigenvalue}

In this section we specialize some results of the previous sections to the cases when $H$ has only one positive eigenvalue and $F=\mathbf{R}$.

Let $F=\mathrm{R}$ and $n \geq 2$, and let $H$ have exactly one positive eigenvalue. With no loss of generality, we take $H=\operatorname{diag}(1,-1, \cdots,-1)$. Then the set

$$
\mathcal{C}=\left\{x=\left(x_{1}, \cdots, x_{n}\right) \in \mathbf{R}^{n_{n}}:[x, x] \geq 0, x_{1} \geq 0\right\}
$$

is a positive cone in $\mathbf{R}^{n}$, i.e., $u+v \in \mathcal{C}$ and $\lambda u \in \mathcal{C}$ whenever $u, v \in \mathcal{C}$ and $\lambda \in[0, \infty)$. The cone $\mathcal{C}$ is called the ice-cream cone. We refer the reader to [LS] for more information about 
this cone, and to $[\mathrm{BP}]$, Section 1.3, and $[\mathrm{Kr}]$ for the properties of the ice-cream cone that follow from the Perron-Frobenius theory; see also Section 4 in [BMRRR2].

Obviously, the set of $H$-nonnegative vectors is given by $\mathcal{C} \cup-\mathcal{C}$. Thus $X \in \mathcal{P}$ if and only if $X(\mathcal{C} \cup-\mathcal{C}) \subseteq \mathcal{C} \cup-\mathcal{C}$.

The following result has been established in [BMRRR2].

THEOREM 4.1. Let $F=\mathbf{R}$ and $H=\operatorname{diag}(1,-1, \cdots,-1)$. Then a real $n \times n$ matrix $X=\left[X_{i j}\right]_{i, j=1}^{n}$ satisfies $X(\mathcal{C}) \subseteq \mathcal{C}$ if and only if it is a doubly $H$-plus matrix with $X_{11} \geq 0$.

Thus, $\mathcal{D} \mathcal{P}$ coincides with the set of real $n \times n$ matrices $X$ for which either $X(\mathcal{C}) \subseteq \mathcal{C}$ or $X(\mathcal{C}) \subseteq(-\mathcal{C})$

A characterization of the class $\mathcal{S P}$ in terms of $\mathcal{C}$ is easily given: A matrix $X$ is a strict $H$-plus matrix if and only if $X$ maps $\mathcal{C} \backslash\{0\}$ into either the interior of $\mathcal{C}$ or the interior of $-\mathcal{C}$. Note that the interior of $\mathcal{C}$ is simply $\left\{x=\left(x_{1}, \cdots, x_{n}\right) \in \mathrm{R}^{n}:[x, x]>0, x_{1}>0\right\}$, and analogously for the interior of $-\mathcal{C}$.

Consider an invertible $H$-plus matrix $X$. Then, since $X$ is an $H$-plus matrix, we have $X(\mathcal{C} \cup-\mathcal{C}) \subseteq \mathcal{C} \cup-\mathcal{C}$. The invertibility of $X$ implies that $X(\mathcal{C}) \subseteq \mathcal{C}$ or $X(\mathcal{C}) \subseteq-\mathcal{C}$. Indeed, choose $x \in \mathcal{C}, x \neq 0$. Then either $X x \in \mathcal{C}$ or $X x \in-\mathcal{C}$. Since $\mathcal{C} \backslash\{0\}$ is connected and $X(\mathcal{C} \backslash\{0\}) \subseteq(\mathcal{C} \backslash\{0\}) \cup(-\mathcal{C} \backslash\{0\})$, we obtain that $X(\mathcal{C} \backslash\{0\}) \subseteq \mathcal{C} \backslash\{0\}$ in the former case and $X(\mathcal{C} \backslash\{0\}) \subseteq-\mathcal{C} \backslash\{0\}$ in the latter case.

PROPOSITION 4.2. The closure of the set of invertible $H$-plus matrices coincides with the set of matrices $X$ such that either $X(\mathcal{C}) \subseteq \mathcal{C}$ or $X(\mathcal{C}) \subseteq-\mathcal{C}$.

Consequently the closure of $\mathcal{S P}$, as well as the closure of $\mathcal{S} \mathcal{P}$, is $\mathcal{D} \mathcal{P}$.

Proof. In view of the remark before the statement of the proposition, we only need to show that $X(\mathcal{C}) \subseteq \mathcal{C}$ or $X(\mathcal{C}) \subseteq-\mathcal{C}$ implies that $X$ can be approximated by invertible $H$-plus matrices. If $X(\mathcal{C}) \subseteq \mathcal{C}$, then $X+\epsilon I, \epsilon>0, \epsilon \downarrow 0$ is such an approximation. If $X(\mathcal{C}) \subseteq-\mathcal{C}$, then $X-\epsilon I, \epsilon>0, \epsilon \downarrow 0$ is such an approximation.

Theorem 2.2 implies that invertible $H$-plus matrices are strictly $H$-plus. In other words, in view of Theorem 4.1 we can approximate every doubly $H$-plus matrix by strictly $H$-plus matrices. Hence, if $F=\mathbf{R}$ and $H$ has exactly one positive eigenvalue, the conjecture stated in the preceding section is true, i.e., the closure of $\mathcal{S P}$ is $\mathcal{D P}$. For the closure of $\mathcal{S P}$ see the remark at the end of section 3 .

We finish this section with a few remarks concerning connectedness.

PROPOSITION 4.3. The set $\mathcal{S P}$ has two connected components, being $\{X \mid X(\mathcal{C} \backslash$ $\{0\}) \subseteq \operatorname{int} \mathcal{C}\}$ and $\{X \mid X(\mathcal{C} \backslash\{0\}) \subseteq-\operatorname{int} \mathcal{C}\}$

Proof. One easily sees that the set of matrices $X$ for which $X(\mathcal{C} \backslash\{0\}) \subseteq$ int $\mathcal{C}$ is itself a convex cone, and hence is connected. Likewise for the set of matrices $X$ for which $X(\mathcal{C} \backslash\{0\}) \subseteq-$ int $\mathcal{C}$. Therefore in view of the characterization of the set $\mathcal{S P}$ given above, $\mathcal{S P}$ has at most two connected components. It remains to show that these two sets are indeed not connected. Suppose the contrary. Then there exist $X \in \mathcal{S P}$, a sequence $Y_{m} \in \mathcal{S P}$ and a sequence $Z_{m} \in \mathcal{S P}$ such that $Y_{m} \rightarrow X, Z_{m} \rightarrow X$, and $Y_{m}$ (resp. $Z_{m}$ ) maps the set $\mathcal{C} \backslash\{0\}$ into int $\mathcal{C}$ (resp. into - int $\mathcal{C}$ ). By continuity, $X$ maps $\mathcal{C}$ into the closure of the interior of $\mathcal{C}$, which is equal to $\mathcal{C}$. By the same token, $X(\mathcal{C}) \subseteq-\mathcal{C}$. Thus, $X(\mathcal{C}) \subseteq \mathcal{C} \cap(-\mathcal{C})=\{0\}$, a contradiction with $X \in \mathcal{S P}$. 
We conclude with a simple observation that the sets $\mathcal{P}$ and $\mathcal{D} \mathcal{P}$ are connected (this observation applies also to the complex case and to the cases when $H$ has more than one positive eigenvalue). Indeed, any $X \in \mathcal{P}$ is connected to zero, which belongs to $\mathcal{P}$, by $t X$, $0 \leq t \leq 1$, and analogously for any $X \in \mathcal{D} \mathcal{P}$.

\section{References}

[AI]

T. Ja. Azizov and E. I. Iohvidov. The development of some of V. P. Potapov's ideas in the geometric theory of operators in spaces with indefinite metric. in: Matrix and Operator Valued Functions OT 72 , eds. 1. Gohberg and L.A. Sakhnovich, Birkhäuser, Basel, 1994, 17-27.

[Ba] G. P. Barker. Theory of cones, Linear Algebra and its Applications 39 (1981), 263-291.

[BG1] A. Ben Artzi and I. Gohberg. On contractions in spaces with an indefinite metric: $G$-norms and spectral radii, Integral Equations and Operator Theory 24 (1996), 422-469.

[BG2] A. Ben Artzi and I. Gohberg. Singular numbers of contractions in spaces with an indefinite metric, Preprint.

[BP] A. Berman and R. J. Plemmons. Nonnegative Matrices in the Mathematical Sciences, Academic Press, New York, 1979; also: SIAM, Philadelphia, 1994.

[Bo] J. Bognár. Indefinite Inner Product Spaces, Springer, Berlin, 1974.

[BMRRR1] Y. Bolshakov, C. V. M. van der Mee, A. C. M. Ran, B. Reichstein, and L. Rodman. Polar decompositions in finite dimensional indefinite scalar product spaces: General theory, to appear in Linear algebra and its Applications.

[BMRRR2] Y. Bolshakov, C. V. M. van der Mee, A. C. M. Ran, B. Reichstein, and L. Rodman. Polar decompositions in finite dimensional indefinite scalar product spaces: Special cases and applications, Operator Theory: Advances and Applications, 87, (I. Gohberg, P. Lancaster, P. N. Shivakumar, eds.), Birkhäuser, Basel, 1996, pp. 61-94; Errata, to appear in Integral Equations and Operator Theory.

[BR] Y. Bolshakov and B. Reichstein. Unitary equivalence in an indefinite scalar product: An analogue of singular value decomposition, Linear Algebra and its Applications, 222 (1995), 155-226.

[Br] M. L. Brodskii. On properties of an operator mapping the non-negative part of a space with indefinite metric into itself, Uspehi Math. Nauk, 14 (1959), 147-152. [Russian]; MR 21, \#5145.

[F] H. Flanders. Elementary divisors of $A B$ and BA, Proc. Amer. Math. Soc. 2 (1951), 871-874.

[Gi1] Ju. P. Ginzburg. On $J$-nonexpansive operator functions, Dokl. Akad. Nauk SSSR 117, No 2 (1957), 171-173 [Russian].

[Gi2] Ju. P. Ginzburg. On $J$-nonexpansive operators in a Hilbert space, Nauchnye Zap. Fak. Fiziki i Matematiki, Odesskogo Gosud. Pedagog. Instituta 22, No 1 (1958), 13-20 [Russian]. 
[GLR] I. Gohberg, P. Lancaster, and L. Rodman. Matrices and Indefinite Scalar Products, OT8, Birkhäuser, Basel, 1983.

[IKL] I. S. Iohvidov, M. G. Krein, and H. Langer. Introduction to Spectral Theory of Operators in Spaces with an Indefinite Metric, "Math. Forschung," Akademie Verlag, Berlin, 1981.

[KS1] M. G. Krein and Ju. L. Shmul'jan. On plus operators in a space with an indefinite metric, Mat. Issled. 1, No. 2 (1966), 131-161 [Russian]. Translated in English: AMS Translations, Series 2, 85 (1969), 93-113.

[KS2] M. G. Krein and Ju. L. Shmul'jan. $J$-polar representation of plus operators, Mat. Issled. 1, No. 2 (1966), 172-210 [Russian]; English Translation: AMS Translations, Series 2, 85 (1969), 115--143.

[Kr] M. A. Krasnoselskii. Positive Solutions of Operator Equations, Noordhoff, Groningen, 1964. [Translated from Russian].

[LS] R. Loewy and H. Schneider. Positive operators on the $n$-dimensional ice cream cone, J. Math. Anal. Appl. 49 (1975), 375-392.

[M] C. V. M. van der Mee. An eigenvalue criterion for matrices transforming Stokes parameters, J. Math. Phys. 34 (1993), 5072-5088.

[MH] C. V. M. van der Mee and J. W. Hovenier. Structure of matrices transforming Stokes parameters, J. Math. Phys. 33 (1992), 3574-3584.

[P1] V. P. Potapov. Multiplicative structure of $J$-nonexpansive matrix functions. Trudy Mosk. Math. Ob. 4 (1955), 125-236 [Russian]; English Translation: AMS Translations, Series 2, 15 (1960), 131-243.

[P2] V. P. Potapov. A theorem on the modulus, I. Main concepts. The modulus. Theory of Functions, Functional Analysis and its Applications 38 (1982), 91101, 129, Kharcov [Russian]. Translated in English: AMS Translations, Series 2, Vol. 138, 55-65. MR 84d:15026

[T] R. C. Thompson. Pencils of complex and real symmetric and skew matrices, Linear Algebra and its Applications 147 (1991), 323-371.

Cornelis V.M. van der Mee

Dipartimento di Matematica

Università di Cagliari

Via Ospedale 72, 09124 Cagliari, Italy

cornelis@vaxca2.unica.it

André C.M. Ran

Faculteit Wiskunde en Informatica

Vrije Universiteit Amsterdam

De Boelelaan 1081

1081 HV Amsterdam, The Netherlands

ran@cs.vu.nl

AMS subject classification 47B50, secondary: 15A21
Leiba Rodman

Department of Mathematics

The College of William and Mary

Williamsburg, VA 23187-8795, USA

lxrodm@math.wm.edu 